\section{LECCIONES APRENDIDAS DEL ESTUDIO DE 10.000 PACIENTES CON SARCOMA DE PARTES BLANDAS. Brennan MF, et al. Ann Surg 2014;260:416-22}

Los autores, del Memorial Sloan Kettering Cancer Center, Nueva York, analizan la información referente a 10.000 pacientes con un sarcoma de partes blandas (SPB) atendidos en ese Centro desde el año 1982. Comienzan refiriéndose al hecho que los SPB representan al menos 80 diferentes variedades de tumores potencialmente malignos de los cuales la mitad son malignos y metastizan y el otro $50 \%$ es localmente agresivo; pero que por su relativa baja frecuencia, no permiten lograr una acabada comprensión de su naturaleza y evolución. Sólo incluyeron prospectivamente pacientes con tumores primarios, localmente recurrentes o con metástasis pero que tuvieron algún procedimiento quirúrgico en la institución, lo que permitió el seguimiento, dado el hecho que se pueden observar recurrencias tardías, décadas más tarde del tratamiento inicial.

Se estudiaron factores de riesgo asociados a los SPB y se hicieron análisis genéticos y moleculares según necesidad, además de reformular los subtipos histológicos que precisarán mejor la biología de los tumores. No obstante, el foco estuvo en analizar la distribución y evolución de acuerdo a la localización original del tumor, su tamaño y profundidad y el grado e histología.

Observaron que el $40 \%$ de los primarios se originó en las extremidades y $38 \%$ era visceral o retroperitoneal. La localización era determinante de la evolución y patrón de recurrencia; las lesiones retroperitoneales e intra-abdominales tienen casi un $50 \%$ de recurrencia a 5 años, después de lo cual progresan lentamente. Las lesiones viscerales y de extremidades tienen recurrencias de $20 \%$ a $25 \%$ a 10 años, pero en ambos casos siguen ocurriendo recurrencias hasta dos décadas después del diagnóstico inicial. Las lesiones de extremidades, con esa recurrencia $(25 \%)$ tienen una prevalencia de muerte específica por la enfermedad del $40 \%$, lo que refleja la importancia de la enfermedad sistémica como causa de muerte. Las lesiones retroperitoneales e intra-abdominales con una mucho mayor recurrencia local, de hasta $60 \%$, tienen un patrón de sobrevida similar al de la recurrencia, con una menor contribu- ción de enfermedad metastásica. Las lesiones viscerales, con recurrencias de menos del $20 \%$ a 10 años, tiene una sobrevida de alrededor del $35 \%$, debido a la enfermedad sistémica. Es decir, la muerte para lesiones viscerales y de extremidades es causa sistémica mientras que para aquellas retroperitoneales e intra-abdominales la causa de muerte se relaciona a la progresión local.

También son importantes predictores el subtipo histológico y el grado. Sarcomas como el sinovial, rabdomiosarcoma y sarcoma de Ewing son siempre clasificados como de alto grado. En extremidades, la recurrencia local es similar $(20 \%)$ ya se trate de un tumor de alto o bajo grado, pero la sobrevida está marcada por el grado; existe recurrencia sistémica en menos del $10 \%$ a 20 años para sarcomas de bajo grado pero para las de alto grado la mortalidad por recurrencia sistémica se eleva al $40 \%$ a 10 años. Han notado que lesiones de bajo grado recurren sistémicamente con características de alto grado.

El tamaño y profundidad, características que no son necesariamente independientes, también influyen en la evolución. Pacientes con lesiones mayores de $10 \mathrm{~cm}$ tienen una sobrevida menor al $40 \%$ a 15 años, observándose un poderoso impacto del tamaño independiente de la profundidad cada vez que el tumor alcanza los $5 \mathrm{~cm}$.

También se observó relación entre sitio del tumor primario y sitio de metástasis; hay dominancia del pulmón para primarios de extremidades y predilección del pulmón para leimiosarcomas uterinos, a diferencia de la relación de los tumores GIST y el hígado. Esto tiene obvias implicancias para el seguimiento de los pacientes. Por último, la metástasis de linfonodos son raras en los SPB, pero si las hay, en ausencia de otras metástasis algunos pacientes se pueden rescatar. Es signo de mal pronóstico la combinación de metástasis de linfonodos y otras metástasis.

Los autores concluyen con una exhaustiva revisión de aspectos moleculares de estos tumores, que pueden influir en las terapias específicas que deben estar orientadas por tumores específicos y no por órganos específicos. Expresan que se pueden concebir lesiones diferentes en distintos sitios del cuerpo con tumores de apariencia histológica distinta pero con vías de progresión y crecimiento que pueden ser inhibidos independiente del sitio de origen del tumor. 